\title{
On the dynamics of the AB Doradus system
}

\author{
J. C. Guirado ${ }^{1}$, I. Martí-Vidal ${ }^{1}$, J. M. Marcaide ${ }^{1}$, L. M. Close ${ }^{2}$, J. C. Algaba ${ }^{1}$, W. Brandner ${ }^{3}$, J.-F. Lestrade ${ }^{4}$, \\ D. L. Jauncey ${ }^{5}$, D. L. Jones ${ }^{6}$, R. A. Preston ${ }^{6}$, and J. E. Reynolds ${ }^{5}$
}

1 Departamento de Astronomía y Astrofísica, Universidad de Valencia, 46100 Burjassot, Valencia, Spain
e-mail: jose.c.guirado@uv.es
2 Steward Observatory, University of Arizona, Tucson, Arizona 85721, USA
3 Max-Planck Institut für Astronomie, Königstuhl 17, 69117 Heidelberg, Germany
4 Observatoire de Paris/LERMA, Rue de l'Observatoire 61, 75014 Paris, France
5 Australian Telescope National Facility, P.O. Box 76, Epping, NSW 2121, Australia
6 Jet Propulsion Laboratory, California Institute of Technology, 4800 Oak Grove Drive, Pasadena, California 91109, USA

Received 4 July 2005 / Accepted 21 September 2005

\section{ABSTRACT}

We present an astrometric analysis of the binary systems AB Dor A/AB Dor C and AB Dor Ba/AB Dor Bb. These two systems of well-known late-type stars are gravitationally associated and they constitute the quadruple AB Doradus system. From the astrometric data available at different wavelengths, we report: (i) a determination of the orbit of $\mathrm{AB}$ Dor $\mathrm{C}$, the very low mass companion to $\mathrm{AB}$ Dor $\mathrm{A}$, which confirms the mass estimate of $0.090 M_{\odot}$ reported in previous works; (ii) a measurement of the parallax of AB Dor Ba, which unambiguously confirms the long-suspected physical association between this star and $\mathrm{AB}$ Dor $\mathrm{A}$; and (iii) evidence of orbital motion of $\mathrm{AB}$ Dor $\mathrm{Ba}$ around $\mathrm{AB}$ Dor $\mathrm{A}$, which places an upper bound of $0.4 M_{\odot}$ on the mass of the pair AB Dor Ba/AB Dor Bb (50\% probability). Further astrometric monitoring of the system at all possible wavelengths would determine with extraordinary precision the dynamical mass of its four components.

Key words. astrometry - stars: kinematics - stars: binaries: close - stars: late-type - stars: individual: Rst 137 B - stars: individual: AB Dor

\section{Introduction}

Astrometry, one of the most classical astronomical disciplines, provides unambiguous mass estimates of celestial bodies via observations of the orbits of binary or multiple systems, as projected on the sky (see, e.g., Kovalevsky 1995). The precise determination of stellar masses is fundamental in astronomy, as this parameter is the primary input to test stellar evolutionary models that provide widely used mass-luminosity relations. In particular, the calibration of the mass-luminosity relation for the lower end of the main sequence is of special interest, since it permits the derivation of the physical properties of very-lowmass (VLM) stars and substellar objects. However, a modelindependent measurement of the mass of these objects is a most demanding task that requires the astrometric follow-up of VLM stars in binary systems (e.g. Lane et al. 2001; Bouy et al. 2004; Golimowski et al. 2004; Close et al. 2005). One of the few VLM objects with astrometric detections is AB Dor C, the companion to AB Dor A.

AB Dor A $(=$ HD 36705) is an active K1 star only 14.9 pc away from the Sun. Due to its ultrafast rotation (0.514 days; Innis et al. 1985), AB Dor A is a strong emitter at all wavelengths, and it has been extensively observed from radio to X-rays (Lim et al. 1992; Mewe et al. 1996; Vilhu et al. 1998; Güdel et al. 2001). AB Dor A possesses a low-mass companion, $\mathrm{AB}$ Dor $\mathrm{C}$, which induces a reflex motion first detected by very-long-baseline-interferometry (VLBI) and the Hipparcos satellite (Guirado et al. 1997). Recently, Close et al. (2005) (CLG) obtained a near-infrared image of $\mathrm{AB}$ Dor $\mathrm{C}$, providing the first dynamical calibration of the mass-luminosity relation for low mass, young objects. AB Dor A has another physical companion, AB Dor B (=Rossiter 137 B, =Rst $137 \mathrm{~B}$ ), a dM4e star, which is also a rapid rotator with a 0.38 day period and is separated from AB Dor A by 9" (Lim 1993). Based on their young age (CLG), common proper motions, and common radial velocities (Innis et al. 1986), it is believed that both stars may be associated. In turn, $\mathrm{CLG}$ found $\mathrm{AB}$ Dor $\mathrm{B}$ to be a tight binary ( $\mathrm{AB}$ Dor $\mathrm{B}=\mathrm{AB}$ Dor $\mathrm{Ba}$ and $\mathrm{AB}$ Dor $\mathrm{Bb}$ ).

$\mathrm{AB}$ Dor $\mathrm{C}$ is the first calibration point for evolutionary tracks in the young VLM regime. From comparison with theoretical predictions, CLG found that the dynamical mass of AB Dor $\mathrm{C}$ is almost twice than predicted by evolutionary models (Chabrier et al. 2000), which suggests that models tend to underpredict the mass of young VLM objects. In this context, a precise estimate of the dynamical mass of $\mathrm{AB}$ Dor $\mathrm{C}$ is extremely important. In this paper we report the details of an improved method to determine the mass of $\mathrm{AB}$ Dor $\mathrm{C}$, which confirms the value of $0.090 M_{\odot}$ given by CLG. We also report on the sky motion of $\mathrm{AB}$ Dor $\mathrm{Ba}$, which shows a nearly-identical 
Table 1. Compilation of all available astrometric data for the AB Doradus system.

\begin{tabular}{|c|c|c|c|c|}
\hline \multicolumn{5}{|c|}{$\mathrm{AB}$ Dor $\mathrm{A}$} \\
\hline Epoch & Instrument & $\alpha(\mathrm{J} 2000)$ & $\delta(\mathrm{J} 2000)$ & Reference \\
\hline 1990.3888 & Hipparcos & $5^{\mathrm{h}} 28^{\mathrm{m}} 44^{\mathrm{s}} .77474 \pm 0.00026$ & $-65^{\circ} 26^{\prime} 56^{\prime \prime} 2416 \pm 00^{\prime \prime} 0007$ & (1) \\
\hline 1990.5640 & Hipparcos & $5^{\mathrm{h}} 28^{\mathrm{m}} 44^{\mathrm{s}} .78652 \pm 0.00025$ & $-65^{\circ} 26^{\prime} 56^{\prime \prime} 2272 \pm 00^{\prime \prime} 0007$ & (1) \\
\hline 1991.0490 & Hipparcos & $5^{\mathrm{h}} 28^{\mathrm{m}} 44^{\mathrm{s}} .77578 \pm 0.00024$ & $-65^{\circ} 26^{\prime} 56^{\prime \prime} 2615 \pm 00^{\prime \prime} 0007$ & (1) \\
\hline 1991.5330 & Hipparcos & $5^{\mathrm{h}} 28^{\mathrm{m}} 44^{\mathrm{s}} .78942 \pm 0.00025$ & $-65^{\circ} 26^{\prime} 56^{\prime \prime} 0757 \pm 00^{\prime \prime} 0008$ & (1) \\
\hline 1992.0180 & Hipparcos & $5^{\mathrm{h}} 28^{\mathrm{m}} 44^{\mathrm{s}} .78202 \pm 0.00024$ & $-65^{\circ} 26^{\prime} 56^{\prime \prime} 1160 \pm 00^{\prime \prime} 0009$ & (1) \\
\hline 1992.2329 & VLBI & $5^{\mathrm{h}} 28^{\mathrm{m}} 44^{\mathrm{s}} .77687 \pm 0.00019$ & $-65^{\circ} 26^{\prime} 56^{\prime \prime} 0049 \pm 0.0^{\prime \prime} 0007$ & (1) \\
\hline 1992.6849 & VLBI & $5^{\mathrm{h}} 28^{\mathrm{m}} 44^{\mathrm{s}} .80124 \pm 0.00018$ & $-65^{\circ} 26^{\prime} 55^{\prime \prime} 9395 \pm 00^{\prime \prime} 0006$ & (1) \\
\hline 1993.1233 & VLBI & $5^{\mathrm{h}} 28^{\mathrm{m}} 44.78492 \pm 0.00024$ & $-65^{\circ} 26^{\prime} 55^{\prime \prime} 9137 \pm 00^{\prime \prime} 0008$ & (1) \\
\hline 1994.8137 & VLBI & $5^{\mathrm{h}} 28^{\mathrm{m}} 44^{\mathrm{s}} .81768 \pm 0.00019$ & $-65^{\circ} 26^{\prime} 55^{\prime \prime} 6866 \pm 00^{\prime \prime} 0005$ & (1) \\
\hline 1995.1425 & VLBI & $5^{\mathrm{h}} 28^{\mathrm{m}} 44.80247 \pm 0.00027$ & $-65^{\circ} 26^{\prime} 55^{\prime \prime} 6248 \pm 00^{\prime \prime} 0011$ & (1) \\
\hline 1996.1507 & VLBI & $5^{\mathrm{h}} 28^{\mathrm{m}} 44^{\mathrm{s}} .81137 \pm 0.00013$ & $-65^{\circ} 26^{\prime} 55^{\prime \prime} 4852 \pm 00^{\prime \prime} 0003$ & (1) \\
\hline 1996.3607 & VLBI & $5^{\mathrm{h}} 28^{\mathrm{m}} 44^{\mathrm{s}} .81776 \pm 0.00018$ & $-65^{\circ} 26^{\prime} 55^{\prime \prime} 3785 \pm 00^{\prime \prime} 0010$ & (1) \\
\hline \multicolumn{5}{|c|}{$\mathrm{AB}$ Dor Ba $(=$ Rst $137 \mathrm{~B})$} \\
\hline 1992.2329 & VLBI & $5^{\mathrm{h}} 28^{\mathrm{m}} 44.39520 \pm 0.0007$ & $-65^{\circ} 26^{\prime} 47^{\prime \prime} 0676 \pm 00^{\prime \prime} 0024$ & (2) \\
\hline 1992.6849 & VLBI & $5^{\mathrm{h}} 28^{\mathrm{m}} 44^{\mathrm{s}} .41973 \pm 0.0006$ & $-65^{\circ} 26^{\prime} 47^{\prime \prime} 0047 \pm 0 .^{\prime \prime} 0021$ & (2) \\
\hline 1993.1233 & VLBI & $5^{\mathrm{h}} 28^{\mathrm{m}} 44.40441 \pm 0.0008$ & $-65^{\circ} 26^{\prime} 46^{\prime \prime} 9869 \pm 00^{\prime \prime} 0028$ & (2) \\
\hline 1994.8137 & VLBI & $5^{\mathrm{h}} 28^{\mathrm{m}} 44^{\mathrm{s}} .43687 \pm 0.0007$ & $-65^{\circ} 26^{\prime} 46^{\prime \prime} 5528 \pm 00^{\prime \prime} 0018$ & (2) \\
\hline 1996.1507 & VLBI & $5^{\mathrm{h}} 28^{\mathrm{m}} 44^{\mathrm{s}} .42842 \pm 0.0005$ & $-65^{\circ} 26^{\prime} 46^{\prime \prime} 5773 \pm 00^{\prime \prime} 0010$ & (2) \\
\hline \multicolumn{5}{|c|}{ Relative Position AB Dor A - AB Dor Ba } \\
\hline Epoch & Instrument & Separation & $\mathrm{PA}\left({ }^{\circ}\right)$ & Reference \\
\hline 1929 & - & $10 . .0$ & 339 & $(3)$ \\
\hline 1985.7 & AAT & $9 . .3 \pm 0 . .3$ & $344 \pm 5$ & (4) \\
\hline 1993.84 & ATCA & $8 . \prime 90 \pm 0 . .02$ & $345.2 \pm 0.1$ & $(5)$ \\
\hline 1994.2 & Dutch/ESO & $8 .{ }^{\prime \prime} 9 \pm 0 .{ }^{\prime} 1$ & $344.7 \pm 0.3$ & (6) \\
\hline 2004.093 & VLT/NACO & $9 . \prime 01 \pm 0 . \prime 01$ & $345.9 \pm 0.3$ & (7) \\
\hline \multicolumn{5}{|c|}{ Relative Position AB Dor A - AB Dor C } \\
\hline Epoch & Instrument & Separation & $\mathrm{PA}\left({ }^{\circ}\right)$ & Reference \\
\hline 2004.093 & VLT/NACO & $0 .{ }^{\prime \prime} 156 \pm 0 . .^{\prime \prime} 010$ & $127 \pm 1^{\circ}$ & $(7)$ \\
\hline \multicolumn{5}{|c|}{ Relative Position AB Dor Ba - AB Dor Bb } \\
\hline Epoch & Instrument & Separation & $\mathrm{PA}\left({ }^{\circ}\right)$ & Reference \\
\hline 2004.098 & VLT/NACO & $0 .{ }^{\prime \prime} 062 \pm 0 . \prime 003$ & $236.4 \pm 3.33^{\circ}$ & $(8)$ \\
\hline
\end{tabular}

(1) Guirado et al. (1997); (2) this paper; (3) Jeffers et al. (1963); (4) Innis et al. (1986); (5) J. Lim, personal communication; (6) Martín \& Brandner (1995); (7) Close et al. (2005); (8) Brandner et al. in preparation.

parallax to that of $\mathrm{AB}$ Dor $\mathrm{A}$ and evidence of the long-term orbital motion around $\mathrm{AB}$ Dor A.

\section{Astrometric data}

In Table 1 we summarize the available astrometric data of the AB Doradus system, which include absolute positions of $\mathrm{AB}$ Dor $\mathrm{A}$ and $\mathrm{AB}$ Dor $\mathrm{Ba}$, relative positions of the 9" pair $\mathrm{AB}$ Dor $\mathrm{A} / \mathrm{AB}$ Dor $\mathrm{Bb}$, and relative positions of the closer pairs $\mathrm{AB}$ Dor $\mathrm{A} / \mathrm{AB}$ Dor $\mathrm{C}$ and $\mathrm{AB}$ Dor $\mathrm{Ba} / \mathrm{AB}$ Dor Bb. New absolute positions of $\mathrm{AB}$ Dor $\mathrm{Ba}$ are presented in this table; they have been obtained from the same VLBI observations that were used to make the astrometric analysis of AB Dor A reported by Guirado et al. (1997). Given the 9" separation, AB Dor A and $\mathrm{AB}$ Dor $\mathrm{Ba}$ lie within the primary beam of each of the telescopes and thus can be observed simultaneously for efficient cancellation of atmospheric systematic errors. The interferometric array has much finer resolution (a few milliarcseconds) and, therefore, the interferometric data for AB Dor Ba could be extracted and processed following the same procedures as described in Sect. 2 of Guirado et al. (1997) for AB Dor A. This in-beam technique is widely used in VLBI observations (e.g. Marcaide \& Shapiro 1983; Fomalont et al. 1999). On the other hand, the relatively low brightness of $\mathrm{AB}$ Dor $\mathrm{Ba}$ $(V=12.6$; Collier Cameron \& Foing 1997) explains the absence of Hipparcos data for this star. In Sect. 3, we revisit the astrometry of the different pairs shown in Table 1.

\section{Astrometric analysis}

\section{1. $A B D$ or $A / A B D$ or $C$ : orbit determination}

The infrared image of $\mathrm{AB}$ Dor $\mathrm{C}$ provided the astrometric data that was used by CLG to constrain the elements of the reflex orbit. The weakness of this procedure was that the relative position $\mathrm{AB}$ Dor $\mathrm{A} / \mathrm{AB}$ Dor $\mathrm{C}$ was not included in the fit, rather it was only used as a discriminator of the orbits that plausibly fit the VLBI/Hipparcos data. In this section, we reestimate the mass of $\mathrm{AB}$ Dor $\mathrm{C}$ using a much improved method that estimates the reflex orbit of $\mathrm{ABDor} A$ by simultaneously combining both the existing VLBI/Hipparcos AB Dor A 
astrometric data and the near-infrared relative position of AB Dor C. Following the classical approach, we modeled the (absolute) position of $\mathrm{AB}$ Dor $\mathrm{A}(\alpha, \delta)$ at epoch $t$ from the expressions:

$$
\begin{aligned}
\alpha(t)=\alpha\left(t_{0}\right)+ & \mu_{\alpha}\left(t-t_{0}\right)+\pi P_{\alpha} \\
& +S_{\alpha}\left(t, X_{1}, X_{2}, X_{3}, X_{4}, P, e, T_{0}\right) \\
\delta(t)=\delta\left(t_{0}\right)+ & \mu_{\delta}\left(t-t_{0}\right)+\pi P_{\delta} \\
& +S_{\delta}\left(t, X_{1}, X_{2}, X_{3}, X_{4}, P, e, T_{0}\right)
\end{aligned}
$$

where $t_{0}$ is the reference epoch, $\mu_{\alpha}, \mu_{\delta}$ are the proper motions in each coordinate, $\pi$ is the parallax, $P_{\alpha}$ and $P_{\delta}$ are the parallax factors (e.g. Green 1985), and $S_{\alpha}$ and $S_{\delta}$ are the reflex orbital motions in $\alpha$ and $\delta$, respectively. The astrometric parameters $\left(\alpha\left(t_{0}\right), \delta\left(t_{0}\right), \mu_{\alpha}, \mu_{\delta}\right.$, and $\left.\pi\right)$ are linear in Eq. (1). The reflex motion, $S_{\alpha}$ and $S_{\delta}$, depends on the seven orbital parameters, namely, $a, i, \omega, \Omega, P, e$, and $T_{0}$. We have used the ThielesInnes coefficients (Green 1985), represented by $X_{1}, X_{2}, X_{3}, X_{4}$, which are defined as combinations of $a, i, \omega, \Omega$. These coefficients behave linearly in Eq. (1), leaving only three non-linear parameters $\left(P, e\right.$, and $\left.T_{0}\right)$ to solve for in our weighted-leastsquares approach.

Since our fitting procedure estimates the orbital parameters of the reflex motion of $\mathrm{AB}$ Dor $\mathrm{A}$, the relative separation $\mathrm{AB}$ Dor $\mathrm{A} / \mathrm{AB}$ Dor $\mathrm{C}$ provided by the infrared data $\left(\Delta \alpha^{\prime}, \Delta \delta^{\prime}\right)$ at epoch $t^{\prime}$ is included in the fit via the corresponding orbital position of the primary star according to the definition of the center of mass of the system:

$$
\begin{aligned}
\Delta \alpha^{\prime} & =-\left(1+q^{-1}\right) S_{\alpha}\left(t^{\prime}\right) \\
\Delta \delta^{\prime} & =-\left(1+q^{-1}\right) S_{\delta}\left(t^{\prime}\right)
\end{aligned}
$$

where $q$ is the mass ratio $m_{\mathrm{c}} / m_{\mathrm{a}}$, with $m_{\mathrm{a}}$ being the mass of the primary and $m_{\mathrm{c}}$ the mass of the companion. The combination of data types in the same fit is reflected in the definition of the $\chi^{2}$ to be minimized:

$$
\begin{aligned}
\chi^{2} & =\sum_{i=1}^{N} \frac{\left(\alpha\left(t_{i}\right)-\widehat{\alpha}\left(t_{i}\right)\right)^{2}}{\sigma_{\alpha}^{2}\left(t_{i}\right)}+\sum_{i=1}^{N} \frac{\left(\delta\left(t_{i}\right)-\widehat{\delta}\left(t_{i}\right)\right)^{2}}{\sigma_{\delta}^{2}\left(t_{i}\right)} \\
& +\left(1+q^{-1}\right)^{2}\left[\frac{\left(S_{\alpha}\left(t^{\prime}\right)-\widehat{S}_{\alpha}\left(t^{\prime}\right)\right)^{2}}{\sigma_{S_{\alpha}}^{2}\left(t^{\prime}\right)}+\frac{\left(S_{\delta}(t)-\widehat{S}_{\delta}\left(t^{\prime}\right)\right)^{2}}{\sigma_{S_{\delta}}^{2}\left(t^{\prime}\right)}\right]
\end{aligned}
$$

where the $\sigma$ 's are the corresponding standard deviations (Table 1) and the circumflexed quantities are the theoretical values of our a priori model. The virtue of the definition of $\chi^{2}$ in Eq. (3) is that the linearity of the orbital parameters is conserved as long as the mass ratio $q$ is not adjusted in the fit. In consequence, $m_{\mathrm{a}}$ is a fixed parameter in our fit (we adopted the value of $0.865 \pm 0.034 M_{\odot}$, as given by CLG). The mass of the secondary $\left(m_{\mathrm{c}}\right)$ will be estimated via an iterative procedure that we outline below:

1. We set a priori values of the three non-linear parameters $(P$, $e$, and $T_{0}$ ). In particular, we sample the following parameter space: $0<P<30$ years, $1990.0<T_{0}<1990.0+P$, and $0<e<1$. To start this procedure, we need an initial value of $m_{\mathrm{c}}$. We take a value of $0.095 M_{\odot}$, which corresponds to the central value of the $m_{\mathrm{c}}$ interval given in Guirado et al. (1997).
Table 2. J2000.0 astrometric and orbital parameters of AB Dor A.

\begin{tabular}{ll}
\hline \hline Parameter & \\
\hline$\alpha^{a}:$ & 52844.7948 \\
$\delta^{a}:$ & -652655.933 \\
$\mu_{\alpha}\left(\mathrm{s} \mathrm{yr}^{-1}\right):$ & $0.0077 \pm 0.0002$ \\
$\mu_{\delta}\left(\operatorname{arcsec} \mathrm{yr}^{-1}\right):$ & $0.1405 \pm 0.0008$ \\
$\pi(\operatorname{arcsec}):$ & $0.0664 \pm 0.0005$ \\
& \\
$P(\mathrm{yr}):$ & $11.76 \pm 0.15$ \\
$a_{1}(\operatorname{arcsec}):$ & $0.0322 \pm 0.0002$ \\
$e:$ & $0.60 \pm 0.04$ \\
$i(\operatorname{deg}):$ & $67 \pm 4$ \\
$\omega(\operatorname{deg}):$ & $109 \pm 9$ \\
$\Omega(\operatorname{deg}):$ & $133 \pm 2$ \\
$T_{o}:$ & $1991.90 \pm 0.04$ \\
& \\
$m_{\mathrm{c}}\left(M_{\odot}\right)^{b}$ & $0.090 \pm 0.003$ \\
\hline
\end{tabular}

${ }^{a}$ The reference epoch is 1993.0. Units of right ascension are hours, minutes, and seconds, and units of declination are degrees, arcminutes, and arcseconds. ${ }^{b}$ Mass range obtained from the period and semimajor axis via Kepler's third law. The mass adopted for the central star AB Dor A was $0.865 \pm 0.034 M_{\odot}$.

2. To find a minimum of $\chi^{2}$, as defined in Eq. (3), we used an iterative method, based on the Brent algorithm (Press et al. 1992). The minimum is defined such that the difference between the reduced- $\chi^{2}$ of two successive iterations is significantly less than unity.

3. From the resulting orbital parameters, we then use Kepler's third law $\left[m_{\mathrm{c}}^{3} /\left(m_{\mathrm{a}}+m_{\mathrm{c}}\right)^{2}=a_{1}^{3} / P^{2}\right.$, with $a_{1}$ the semimajor axis of the reflex orbit] to estimate the mass $m_{\mathrm{c}}$ of AB Dor C.

4. We iterate the least squares fit (step 2) using as a priori values the new set of adjusted orbital parameters, and estimated $m_{\mathrm{c}}$.

5. A final set of orbital parameters is obtained once the estimated $m_{\mathrm{c}}$ is self-consistent, that is, the difference between the value of $m_{\mathrm{c}}$ calculated in step 3 from consecutive sets of adjusted orbital parameters is negligible (i.e. $\ll 0.001 M_{\odot}$ ).

The resulting orbital parameters, and the estimate of the mass of $\mathrm{AB}$ Dor $\mathrm{C}$, are shown in Table 2 and represented in Fig. 1. These values are fully compatible with those given by CLG. However, our method shows the robustness of the determined orbit. Despite the wide range of parameter space investigated, the solution found for the reflex orbit of $\mathrm{AB}$ Dor $\mathrm{A}$ is unique (see Fig. 2). This is a remarkable result: for astrometrically determined orbits, Black \& Scargle (1982) predicted a coupling between the proper motion and the orbital wobble, resulting in an underestimation of the period and semimajor axis. This coupling is present in the VLBI/Hipparcos data that only covers $51 \%$ of the reflex orbit. Our least-squares approach copes partially with this effect, since the astrometric and orbital parameters are estimated simultaneously. However, the VLT/NACO data not only extends significantly the observing time baseline, but represents purely orbital information, independent of proper motion and parallax effects. In practice, the combination of astrometric data of the primary star with astrometric data of 

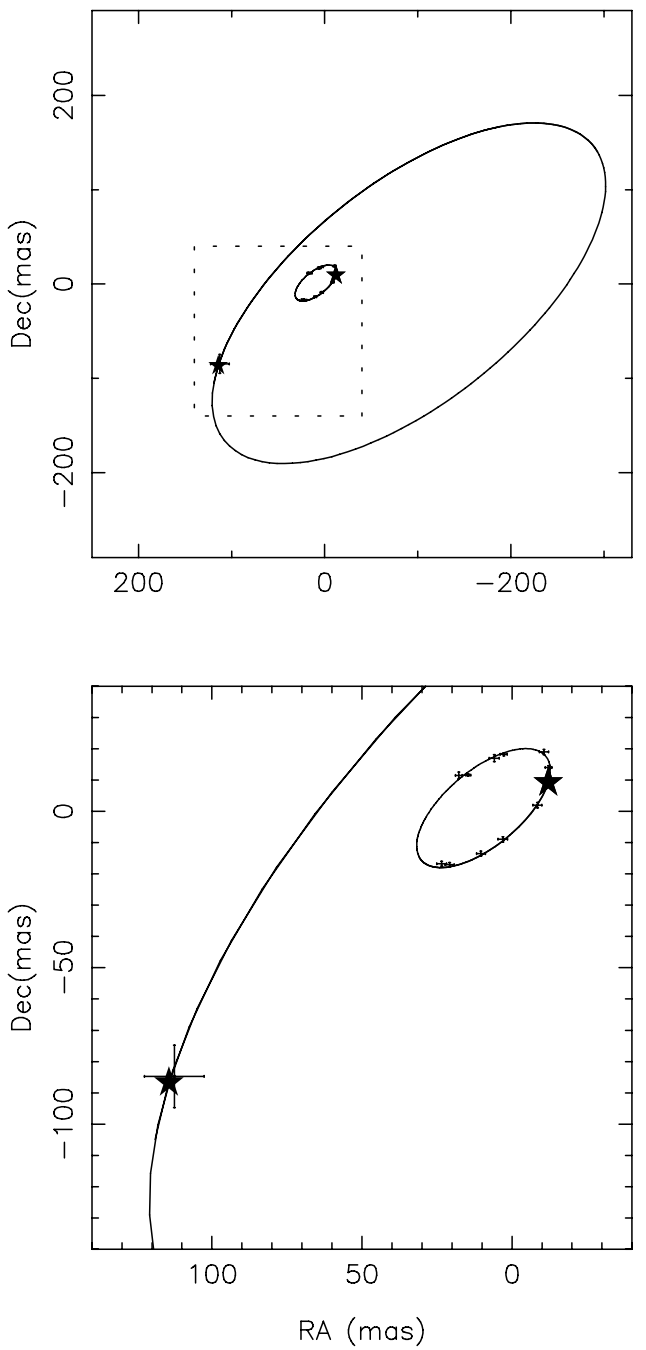

Fig. 1. Above: orbits of the pair $\mathrm{AB}$ Dor $\mathrm{A}$ (inner ellipse) and $\mathrm{AB}$ Dor C (outer ellipse). Below: blow up of the dotted square in the figure above. VLBI and Hipparcos data points are marked in AB Dor A's orbit, while the VLT/NACO AB Dor C position relative to $\mathrm{AB}$ Dor A is indicated in AB Dor C's ellipse. The star symbols over the orbits correspond the astrometric predictions at epoch 2004.093, based on the orbital elements given in Table 2.

the relative orbit improves the fit dramatically, constraining the orbital periods allowed by the astrometric data of the primary only. In our case, the constraint is such that the only allowed period is $11.76 \pm 0.15 \mathrm{yr}$. In general, our results show the combination of different techniques is more effective than any one technique alone.

\subsubsection{Error analysis}

Our least-squares procedure provides formal errors for the adjusted orbital parameters. However, other systematic contributions need to be taken into account. In particular, this includes the uncertainty associated to the mass of $\mathrm{AB}$ Dor $\mathrm{A}$, which is a fixed parameter in our analysis $\left(0.865 \pm 0.034 M_{\odot}\right)$. To estimate this error contribution, we altered the mass of AB Dor A by one standard deviation and repeated the fitting procedure to obtain the change in the orbital parameters and the mass

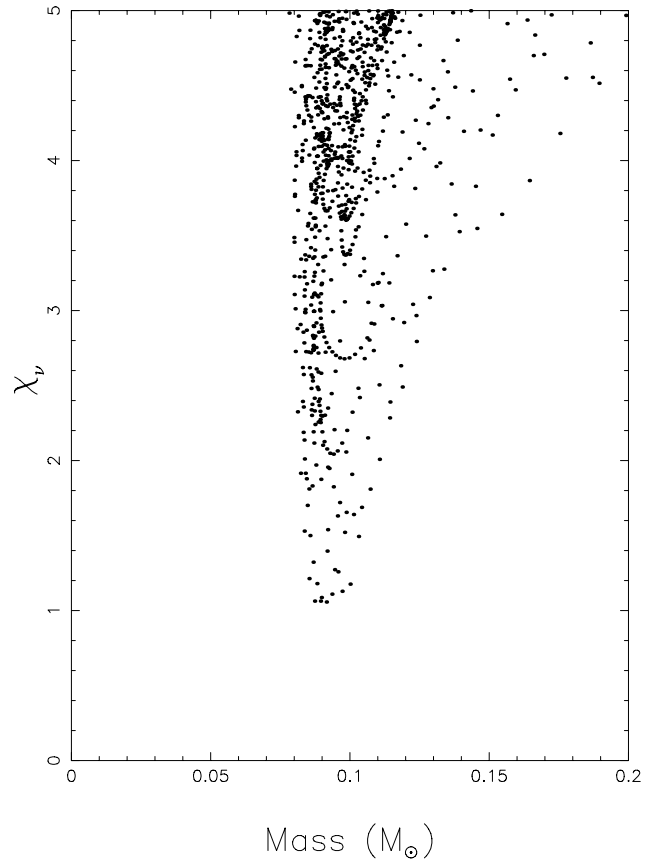

Fig. 2. Result of the exploration of the AB Dor A reflex orbit. A welldefined minimum is found for a mass companion of $0.090 M_{\odot}$. See Sect. 3.1.

of $\mathrm{AB}$ Dor $\mathrm{C}$. We note that this a conservative approach, since this technique fails to account for the correlation of $m_{\mathrm{a}}$ with the rest of the parameters. The resulting parameter changes were added in quadrature with the formal errors of our fit (see Table 2). As expected, the $0.003 M_{\odot}$ standard deviation of the mass of $\mathrm{AB}$ Dor $\mathrm{C}$ is dominated by the uncertainty in the mass of $\mathrm{AB}$ Dor A, while the standard deviations of the rest of the parameters are dominated by the statistical errors.

We also checked the dependence of our results on the choice of the a priori value of $m_{\mathrm{c}}$ in step 1 of our fitting procedure (Sect. 3.1). We found that the results are insensitive to this choice. The postfit residuals of the positions of AB Dor A exhibits an rms of $\sim 1$ mas at each coordinate, consistent with the standard errors, and with no evidence, within uncertainties, of any further orbiting companion to $\mathrm{AB}$ Dor A.

\subsection{VLBI astrometric parameters of $A B D$ or $B a$}

Innis et al. (1985) presented radial velocity measurements of $\mathrm{AB}$ Dor $\mathrm{Ba}$, the 9" companion to AB Dor A. Their measurements do not differ from those of $\mathrm{AB}$ Dor $\mathrm{A}$ within the uncertainties. Additionally, Innis et al (1986) and Martín \& Brandner (1995) reported close agreement between the proper motions of both stars. These results are strong arguments in favor of a physical association of both stars. We used the VLBI (absolute) positions of $\mathrm{AB}$ Dor $\mathrm{Ba}$ given in Table 1 to derive the parallax and proper motion via a least-squares fit. The results of this fit are presented in Table 3, which shows that the parallax of $\mathrm{AB}$ Dor $\mathrm{Ba}$ is coincident with that of $\mathrm{AB}$ Dor $\mathrm{A}$ to within the uncertainties, which provides independent and conclusive evidence for the association of both stars. Comparison of Table 1 and Table 3 shows that the proper motion of $\mathrm{AB}$ Dor $\mathrm{Ba}$ derived 
Table 3. J2000.0 VLBI astrometric parameters of AB Dor Ba.

\begin{tabular}{ll}
\hline \hline Parameter & \\
\hline$\alpha^{1}:$ & $52844.4123 \pm 0.0002$ \\
$\delta^{b}:$ & $-652646.9974 \pm 0.0015$ \\
$\mu_{\alpha}\left(\mathrm{s} \mathrm{yr}^{-1}\right):$ & $0.0085 \pm 0.0002$ \\
$\mu_{\delta}\left(\operatorname{arcsec} \mathrm{yr}^{-1}\right):$ & $0.134 \pm 0.0012$ \\
$\pi(\operatorname{arcsec}):$ & $0.0666 \pm 0.0015$ \\
\hline
\end{tabular}

from the radio data appears significantly different to that of AB Dor A. Given the relatively small uncertainty of our determination, this does not contradict previous (and coarser) measurements of common proper motion. Rather, we interpret this extra proper motion of $\mathrm{AB}$ Dor Ba towards the south-east as a result of the orbital motion around AB Dor A (see Sect. 3.3).

The postfit residuals of $\mathrm{AB}$ Dor $\mathrm{Ba}$ show a systematic signature, both in right ascension and declination, which corresponds to a relatively high $\mathrm{rms}$ of $\sim 4$ mas. The short time span between our separate VLBI observations makes it unlikely that this signature is an effect of the long-term gravitational interaction of $\mathrm{AB}$ Dor Ba with $\mathrm{AB}$ Dor A. Rather, this signature could be assigned to the 0.070 " companion (ABDorBb) of $\mathrm{AB}$ Dor $\mathrm{Ba}$ seen in the VLT/NACO observations reported by CLG. As for the revision of the reflex orbit of $\mathrm{AB}$ Dor $\mathrm{A}$, we attempted to get estimates of the orbital elements of the reflex motion of $\mathrm{AB}$ Dor $\mathrm{Ba}$ by combining the radio data with the VLT relative position between $\mathrm{AB}$ Dor Ba/AB Dor Bb (Table 1). However, our analysis did not yield useful bounds to the mass of this pair, showing that the number of data points is still insufficient and, more likely, they do not properly sample the expected short period of this tight pair.

\section{3. $A B D$ or $A / A B D o r B a$ : evidence of orbital motion of $A B$ Dor $B a$}

As stated in the previous section, evidence of the motion of $\mathrm{AB}$ Dor $\mathrm{Ba}$ around $\mathrm{AB}$ Dor $\mathrm{A}$ can be obtained from the radio data alone. In order to get more precise evidence of this orbital motion, we augmented our data set with relative positions $\mathrm{AB}$ Dor $\mathrm{A} / \mathrm{AB}$ Dor $\mathrm{Ba}$ found in the literature (see Table 1). We then corrected all relative positions $\mathrm{AB}$ Dor $\mathrm{A} / \mathrm{AB}$ Dor $\mathrm{Ba}$ for the reflex orbital motion of $A B$ Dor A (Table 2), effectively referring the positions of $\mathrm{AB}$ Dor $\mathrm{Ba}$ to the center of mass of the AB Dor A/AB Dor C system.

We attempted to constrain the relative orbit of $\mathrm{AB}$ Dor $\mathrm{A} / \mathrm{AB}$ Dor $\mathrm{Ba}$ following a similar analysis to that described in Sect. 3.1, fitting only the 7 parameters of the relative orbit. We sampled all possible periods up to 5000 years and eccentricities from 0 to 1 . We selected as plausible orbits those whose reduced $-\chi^{2}$ differs by $25 \%$ of the minimum. For each plausible orbit, the mass of the complete system was estimated from Kepler's third law, now expressed in terms of the parameters of the relative orbit:

$\frac{(a / \pi)^{3}}{P^{2}}=M_{(\mathrm{A}+\mathrm{C})}+M_{(\mathrm{Ba}+\mathrm{Bb})}$

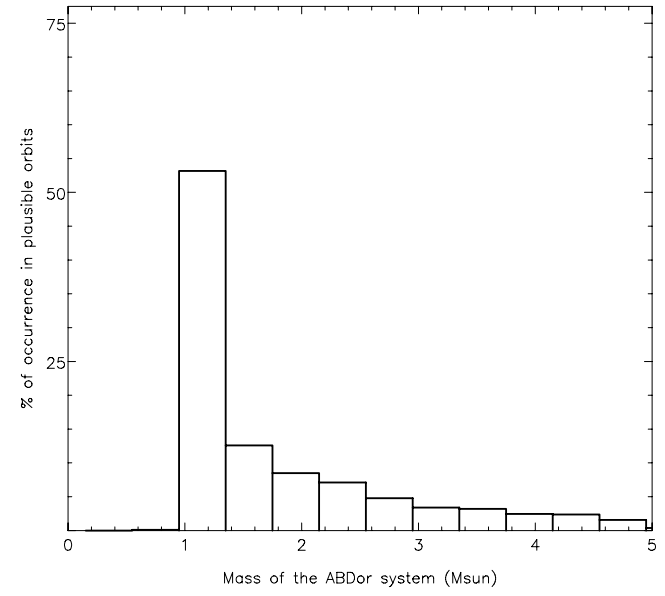

Fig. 3. Histogram of plausible orbits for the relative orbit of $\mathrm{AB}$ Dor $\mathrm{Ba}$ around $\mathrm{AB}$ Dor A. More than $50 \%$ of the plausible orbits correspond to a total mass of the system in the range $0.95-1.35 M_{\odot}$.

where $M_{(\mathrm{A}+\mathrm{C})}$ and $M_{(\mathrm{Ba}+\mathrm{Bb})}$ are the combined masses of $\mathrm{AB}$ Dor $\mathrm{A} / \mathrm{AB}$ Dor $\mathrm{C}$ and $\mathrm{AB}$ Dor $\mathrm{Ba} / \mathrm{AB}$ Dor $\mathrm{Bb}$, respectively, $a$ is the relative semimajor axis ( $\operatorname{arcsec}), \pi$ is the parallax (arcsec; Table 2), and $P$ is the period (yr). The poor coverage of the orbit favors a correlation between the orientation angles and the eccentricity, allowing a wide range of orbital parameters that fit our data equally well. However, a similar correlation between $P$ and $a$ imposes a constraint on the determination of the mass of the system via Eq. (4), which is represented in the histogram of Fig. 3. From the plausible orbits selected, more than $50 \%$ correspond to a total mass of the AB Doradus system in the interval $0.95-1.35 M_{\odot}$ (see Fig. 4 for examples of plausible orbits). Larger masses are not excluded, but the required orbital configurations for masses outside this range occur with significantly reduced probability.

If we assume the total mass of the $\mathrm{AB}$ Doradus system lies in the interval $0.95-1.35 M_{\odot}$, the combination with our estimate of $M_{(\mathrm{A}+\mathrm{C})}\left(0.956 \pm 0.035 M_{\odot}\right.$; see Sect. 1) suggests an upper bound to the mass of the pair $\mathrm{AB}$ Dor $\mathrm{Ba} / \mathrm{AB}$ Dor $\mathrm{Bb}$ of $0.4 M_{\odot}$. This upper limit to $M_{(\mathrm{Ba}+\mathrm{Bb})}$ looks too coarse to calibrate evolutionary models. Nevertheless, it can be transformed into a bound to the age of this pair. To do this, we used the $K$-band 2MASS photometry of AB Dor Ba, and the $K$-band difference between $\mathrm{AB}$ Dor $\mathrm{Ba}$ and $\mathrm{AB}$ Dor Bb reported by CLG. The comparison with Baraffe et al. (1998) isochrones suggests an age for this pair in the range of 50-120 Myr. This range is compatible with previous values of the age of $\mathrm{AB}$ Dor $\mathrm{Ba}$ (30-100 Myr; Collier Cameron \& Foing 1997). However, our age estimate for $\mathrm{AB}$ Dor $\mathrm{Ba} / \mathrm{AB}$ Dor $\mathrm{Bb}$ is not conclusive: first, the masses of the individual components are yet to be determined, and second, there are indications that the evolutionary models might need revision, since they tend to underpredict masses for very young objects below $0.3 M_{\odot}$ (CLG; Reiners et al. 2005).

\section{Summary}

We have revisited the different orbits in the quadruple system in AB Doradus. Paradoxically, this system, where the 

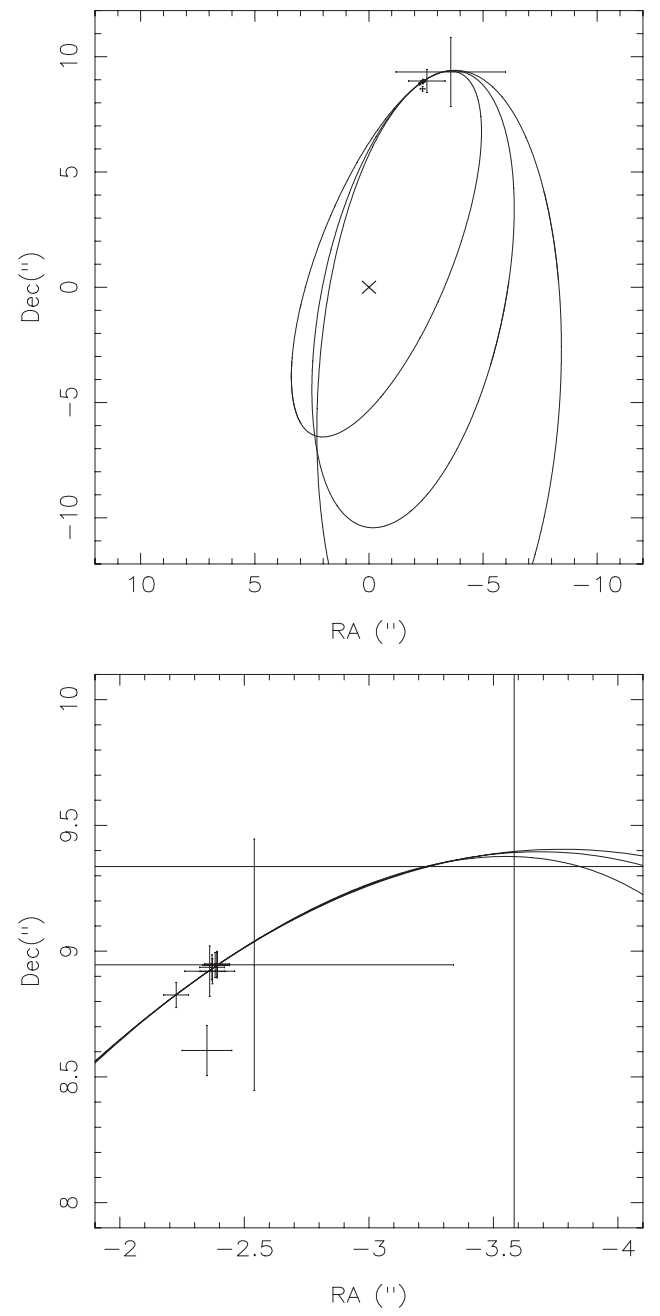

Fig. 4. Above: positions of $\mathrm{AB}$ Dor $\mathrm{Ba}$ with respect to the center of mass of $\mathrm{AB}$ Dor $\mathrm{A} / \mathrm{AB}$ Dor $\mathrm{C}$ (see Table 1) and several allowed orbital solutions. The displayed orbits correspond to a total mass of the system in the range $0.95-1.35 M_{\odot}$ with periods of 1400,2300 , and 4300 years. The cross at the origin indicates the position of AB Dor A/AB Dor C. Below: blow up of the region containing the measurements.

measurement of precise radial velocities is difficult due to the fast rotation of the main components, has become an extraordinary target for astrometric techniques in different bands of the electromagnetic spectrum. From our analysis of the available data, we have re-estimated the mass of the VLM star $\mathrm{AB}$ Dor $\mathrm{C}$ by using a least-square approach that combines the data from radio, optical, and infrared bands. Although the data do not cover a full orbit, the mass and orbital elements of $\mathrm{AB}$ Dor $\mathrm{C}$ are strongly constrained and fully compatible with those reported by CLG. Further monitoring of the reflex orbit of AB Dor A via VLBI observations, and of the relative orbit AB Dor A/AB Dor C via VLT/NACO observations, will result in independent estimates of the masses of the components of this pair. From the absolute radio positions of AB Dor $\mathrm{Ba}$, we have determined the absolute sky motion (i.e. not referred to the motion of $\mathrm{AB}$ Dor $\mathrm{A}$ ) of this star and, in particular, its parallax, which is identical, within the uncertainties, to that of AB Dor A. This confirms the association of both stars.
The mass of $\mathrm{AB}$ Dor $\mathrm{C}$ serves as a precise calibration point for mass-luminosity relations of young VLM stars. Likewise, other components of $\mathrm{AB}$ Doradus may provide new calibration points for slightly higher masses. We have found evidence for the long-term orbital motion of $\mathrm{AB}$ Dor $\mathrm{Ba} / \mathrm{AB}$ Dor $\mathrm{Bb}$ around AB Dor A/AB Dor C. From an exploration of the multiple orbits that fit the available data we find that the most probable mass upper limit of the pair is $0.4 M_{\odot}$. This limit maps into an age range of 50-120 Myr using the isochrones provided by Baraffe et al. (1998). Further monitoring with the appropriate sampling, both in radio and infrared, should provide the orbital elements of both the relative and reflex orbits of the pairs $\mathrm{AB}$ Dor $\mathrm{A} / \mathrm{AB}$ Dor $\mathrm{C}$ and $\mathrm{AB}$ Dor $\mathrm{Ba} / \mathrm{AB} \mathrm{Dor} \mathrm{Bb}$, from which would follow precise, model-independent, estimates of the masses of the four components of this system.

Acknowledgements. This work has been supported by the Spanish DGICYT grant AYA2002-00897. The Australia Telescope is funded by the Commonwealth Government for the operation as a national facility by the CSIRO. Part of this research was carried out at the Jet Propulsion Laboratory, California Institute of Technology, under contract with the US National Aeronautics and Space Administration.

\section{References}

Baraffe, I., Chabrier, G., Allard, F., \& Hauschildt, P. H. 1998, ApJ, 499, 205

Black, D. C., \& Scargle, J. D. 1982, ApJ, 263, 854

Bouy, H., Duchene, G., Köhler, R., et al. 2004, A\&A, 423, 341

Chabrier, G., Baraffe, I., Allard, F., \& Hauschildt, P. H. 2000, ApJ, 542,464

Close, L. M., Lenzen, R., Guirado, J. C., et al. 2005, Nature, 433, 286 (CLG)

Collier Cameron, A., \& Foing, B. 1997, The Observatory, 117, 218

Fomalont, E. B., Goss, W. M., Beasley, A. J., \& Chatterje, S. 1999, AJ, 117, 3025

Golimowski, D. A., Henry, T. J., Krist, J. E., et al. 2004, ApJ, 128, 1733

Green, R. M. 1985, Spherical Astronomy (Cambridge: Cambridge University Press)

Güdel, M., Audard, M., Briggs, K., et al. 2001, A\&A, 365, L336

Guirado, J. C., Reynolds, J. E., Lestrade, J.-F., et al. 1997, ApJ, 490, 835

Innis, J. L., Coates, D. W., Thompson, K., \& Robinson, R. D. 1985, PASAu, 6, 156

Innis, J. L., Thompson, K., \& Coates, D. W. 1986, MNRAS, 223, 183

Jeffers, H. M., van der Bos, W. H., \& Greeby, F. M. 1963, Index Catalogue of Visual Double Stars, Lick Observatory, Mount Hamilton, California

Kovalevsky, J. 1995, Modern Astrometry (Berlin: Springer)

Lane, B. F., Zapatero Osorio, M. R., Britton, M. C., et al. 2001, ApJ, 560,390

Lim, J., Nelson, G. J., Castro, C., et al. 1992, ApJ, 405, L33

Lim, J. 1993, ApJ, 405, L33

Martín, E., \& Brandner, W. 1995, A\&A, 294, 744

Marcaide, J. M., \& Shapiro, I. I. 1983, AJ, 88, 1133

Mewe, R., Kaastra, J. S., White, S. M., \& Pallavicini, R. 1996, A\&A, 315,170

Press, W. H., Teukolsky, S. A., Vetterling, V. T., \& Flannery, B. P. 1992, Numerical Recipes in FORTRAN: The Art of Scientific Computing (Cambridge: Cambridge Univ. Press)

Reiners, A., Basri, G., \& Mohanty, S. 2005, ApJ, in press [arXiv: astro-h/0506501]

Vilhu, O., Muhli, P., Huovelin, J., et al. 1998, ApJ, 115, 1610 